\title{
Technology for new surgical aortic valve replacement: current evidence and future directions
}

\author{
Jeffrey Shuhaiber \\ Department of Cardiothoracic, Heart and Vascular Institute, Baystate Medical Center, Springfield, MA, USA \\ Correspondence to: Jeffrey Shuhaiber MD. Department of Cardiothoracic, Heart and Vascular Institute, Baystate Medical Center, 2 Medical Center \\ Drive, Suite 205, Springfield, MA 01107, USA. Email: Jeffrey.shuhaiber@gmail.com. \\ Comment on: Filip G, Litwinowicz R, Kapelak B, et al. Midterm follow-up after suture-less aortic heart valve implantation. J Thorac Dis 2018. [Epub \\ ahead of print].
}

Submitted Oct 29, 2018. Accepted for publication Nov 05, 2018.

doi: $10.21037 /$ jtd.2018.11.25

View this article at: http://dx.doi.org/10.21037/jtd.2018.11.25

Surgical aortic valve surgery has stood the test of time as a reliable, reproducible, durable and readily available surgery using either mechanical or tissue or autologous valve technology. Performed well, the long-term results are excellent. All new heart valve related trials including catheter based [transcatheter aortic valve replacement (TAVR)] are compared to the conventional surgical aortic valve replacement (C-AVR). So why do we need new valves and techniques to insert aortic valves? What is driving this and why should we adopt them?

The short answer is we have no ideal valve commercially available to this date but we have continued to help our patients through innovative methods. Performing the Donald Ross procedure is a challenging operation to learn and reproduce consistently yet patients who receive a good Ross operation do best. Nearly two decades ago there was a notion in our specialty towards small access aortic valve surgery via a variety of thoracotomy or sternotomy techniques (1). Advantages of small incision surgery if done safely with optimal myocardial protection, arterial perfusion and venous drainage include less surgical trauma, less blood loss and intensive care unit stay with moderate quality of evidence at best $(1,2)$. There is no evidence that small incision access aortic valve surgery has better mortality or reduced cardiopulmonary bypass time or cross clamp. Whilst minimally invasive cardiac surgery is possible and reproducible, why this has not become universal?

The natural evolution of the sutureless aortic valves (SU-AVR) such as $3 \mathrm{~F}$ enable is welcomed. In this series by Dr. Grzegorz Filip from Krakow, Poland of 25 patients with complete follow up underwent isolated SU-AVR implantation. The median preoperative, STS score was $2.96 \pm 2.73$. Preoperatively, $65.4 \%$ of patients were in NYHA class III or IV, the peak/mean gradient transaortic gradient was $84.6 / 52.1 \mathrm{mmHg}$. The authors accomplished a significant and sustainable drop in the transaortic gradient up to 5 years postoperatively ( $<17 \mathrm{mmHg}$ and $<9 \mathrm{mmHg}$ ). The majority of patients were female, average age of 71.6 years. The aortic clamp time was up to 58 minutes (3). They are to be congratulated on excellent clinical outcomes with no deaths, need for pacemaker, endocarditis or thromboembolic complications. They had only one patient during this period with mild paravalvular leak 1 year after implantation. Though it is a small series, it was this group that implanted the first sutureless valve in the world and deserves recognition. Other rapid deployment aortic valves (RD-AVR) such as Intuity (Edwards lifesciences LLC, Irvine, CA, USA) have been fine addition to our fine conventional tissue aortic valves. The similar question also arises—will these new RD and SU-AVR become widely adopted or will they have a unique role?

The reader by now wonders that this editorial provides more questions than answers maybe because that these new valves have shown up at a time when TAVR does not require cardiopulmonary bypass and without any chest incision in most - a far less invasive and readily available procedure.

These new aortic valves (SU-AVR and RD-AVR) have been developed and clinically implanted with overriding premise and promise to reduce the ischemic time to the 
Table 1 Illustrates the extent of the outcome of paravalvular leak, need for PPI, durability, cost, endocarditis and PPM

\begin{tabular}{lccc}
\hline Type of valve & SU-AVR/RD-AVR & TAVR & C-AVR \\
\hline Paravalvular leak & ++ & + & + \\
Need for PPI & ++ & $+++(++$ for combined cases $)$ \\
Track record and follow up & $+++(5$ years $)$ & + & $+++(>15$ years $)$ \\
Cost & +++ & +++ & + \\
Endocarditis & + (short term) & ++ & ++ \\
PPM & + & +++ & + \\
\hline
\end{tabular}

+ , denotes low or mild risk; ++, denotes moderate or increased or intermediate risk; +++, denotes most or highest risk in relative among the different valves stated in Table 1 only. PPI, permanent pacemaker implantation; PPM, patient prosthesis mismatch.

heart, allow easy, effective and safe valve implantation under direct vision $(4,5)$. These valves are rapidly deployed following aortic valve leaflet resection and meticulous annulus decalcification as in conventional AVR. These new surgical valves are built on a stent with either the bovine pericardial tissue (Perceval, LivaNova PLC London), equine pericardial tissue with self-expanding nitinol support frame (3F enable, Medtronic Inc., Minneapolis, MN, USA) and porcine aortic valve tissue sewn on the nitinol stent (Intuity Valve Edwards). Currently in the United States, one clinically approved SU-AVR and one clinically approved RD-AVR is commercially available. The $3 \mathrm{~F}$ enable was the world's first commercially available sutureless tissue heart valve. Of the valve studies published on safety and efficacy with 5 years follow up the valve performed well with $93 \%$ freedom from valve mortality comparable to traditional biological surgical valves (6). Medtronic $3 \mathrm{~F}$ Enable received CE (Conformité Européenne) Mark in December 2009. Based on review of the literature, the $3 \mathrm{~F}$ valve was withdrawn.

Nonetheless, with the prevalence of TAVR, the appeal for new rapid surgical deployment of aortic valves became an attraction not just for the ease of implantation but also to the patient. Two trans-Atlantic Ocean trials recently published have demonstrated advantages beyond short myocardial ischemic times and through a small surgical incision $(4,5)$. These two trials involved the Intuity Valve (Edwards). The two additional advantages include a lower risk of endocarditis at 1 year compared to those with TAVR and SAVR and lower risk of permanent pacemaker implantation (PPI) compared to TAVR $(4,5)$. Improved patient prosthesis mismatch (PPM) compared to TAVR and SAVR (Intuity Valve) $(4,5)$ have been experienced as well. These new benefits extend in my opinion from reducing the bulk of tissue in the aortic root by surgical resection of leaflets and minimize the foreign body in the aortic root with less sutures and pledgets (7). TAVR does not offer leaflet resection or annulus decalcification. Obviously, these rates for PPI, PPM and paravalvular leak vary from one study to another but the overriding massage is these new valves are second best compared to C-AVR. With that being said, TAVR remains a "Wild Card "with a very attractive least invasive method for implantation among all other valves. In the next few paragraphs, I will detail the plausible issues for each valve. For now given the available data, I have listed the degree of severity for C-AVR, SU-AVR/RDAVR and TAVR. By now means this is robust or statistically significant but based on the analysis of the given available multi-institutional data (Table 1).

I could foresee the need to place rapid deployment sutureless valve to save precious operative time, these valves are of value in combined procedures such as double valve surgery or aortic valve and coronary revascularization or in redo surgery. The value of reducing the ischemic time to the myocardium is helpful and more so in a patient with already reduced ejection fraction. However, in a recent German registry trial comparing sutureless valves with outcomes: risk of disabling stroke was higher following implantation of the Intuity than conventional aortic valves (8). While the patients experienced reduced aortic cross clamp times and operative times this did not translate to better hospital survival and stroke. The latter are very important outcomes patients need to know. The analysis involved near 21,000 patients who received conventional valve and 1,125 who received rapid deployment (Intuity and Perceval) as well as the Medtronic's 3 F Enable.

I am sure heart surgeons welcome the addition of the new technology and techniques, though often this is with the critical eye. These valves and surgeons will always go through the learning curve as they get adopted and 
outcomes analyzed. Deliberate practice with room for errors is not a luxury we can tolerate in the current climate of low mortality and morbidity in cardiac surgery. Valve migration, reoperation and durability will determine each valves' fate regardless of the surgeon technical ability. Surgeon-proctoring and mentoring is therefore needed to minimize errors and optimize hospital outcomes. Long term durability at 5 years with good hemodynamic profile as in the $3 \mathrm{~F}$ enable is encouraging though most practicing surgeons would want to wait for more data before making any meaningful conclusion. Currently, the International Valvular Surgery Study Group (30 centers and 10 Countries) aims to potentially provide the best evidence available in long-term follow-up of SU/RD valves with follow up date up to 2022 (9) to affirm the durability of the valve.

While TAVR has taken the most rapid introduction and implementation in recent cardiovascular history with significant diffusion dominating the literature and press, the durability of the valve leaflets housed in the stent remains an unknown long term. Is that important? The mean age for both the high risk and intermediate risk TAVR patients remained high at 81 years of age with limited follow up close to 3 years. However, the mean life expectancy is 79 years in the USA. Application of TAVR in younger patients therefore may not be the right clinical decision to render when surgical valves have had a better track record. Importantly, up to $13 \%$ risk of subclinical leaflet thrombosis has been reported with TAVR (10). We do know that surgical tissue valves among TAVR valves have a lower leaflet thrombosis rate and have much better track record in patients above 65 years of age including those in the geriatric age group. Although, a special 4-dimensional volume-rendered computed tomography scan needs to be done for surgical valves as well. Another disturbing concern surrounds the most recent multi-institutional study upon which a high number of TAVR patients experienced severe and moderate cases of PPM and noted risk for death and heart failure readmission were $19 \%$ and $12 \%$ higher after 1 year (11). Is this the chronobiology of the patient influencing the valve function or is it the TAVR valve misbehaving after crimping or both?

Innovation is needed and value needs to be measured contemporarily. There are more answers waiting through future of research trials. These are important components of our duties to the public.

SU-AVR and RD-AVRs are more expensive with no superior demonstrable impact on patient outcomes yet especially with stroke or survival. This does not diminish their role but can be utilized in specific circumstances when the aortic root is hostile due to aortic annulus scarring as in repeat cardiac surgery. Future trials akin to TAVR randomizing CABG/AVR patients for SAVR versus SUAVR/RD-AVR maybe a starting point. Time is on our side and order of evidence presenting in time may allow each valve attain a unique role tailored to each patient.

\section{Acknowledgements}

None.

\section{Footnote}

Conflicts of Interest: The author has no conflicts of interest to declare.

\section{References}

1. Kirmani BH, Jones SG, Malaisrie SC, et al. Limited versus full sternotomy for aortic valve replacement. Cochrane Database Syst Rev 2017;4:CD011793.

2. Vola M, Fuzellier JF, Campisi S, et al. Closed chest human aortic valve removal and replacement: Technical feasibility and one year follow-up. Int J Cardiol 2016;211:71-8.

3. Filip G, Litwinowicz R, Kapelak B, et al. Midterm followup after suture-less aortic heart valve implantation. J Thorac Dis 2018. [Epub ahead of print].

4. Barnhart GR, Accola KD, Grossi EA, et al. TRANSFORM (Multicenter Experience With Rapid Deployment Edwards INTUITY Valve System for Aortic Valve Replacement) US clinical trial: Performance of a rapid deployment aortic valve. J Thorac Cardiovasc Surg 2017;153:241-51.e2

5. Kocher AA, Laufer G, Haverich A, et al. One-year outcomes of the Surgical Treatment of Aortic Stenosis With a Next Generation Surgical Aortic Valve (TRITON) trial: a prospective multicenter study of rapid-deployment aortic valve replacement with the EDWARDS INTUITY Valve System. J Thorac Cardiovasc Surg 2013;145:110-5; discussion 115-6.

6. Englberger L, Carrel TP, Doss M, et al. Clinical performance of a sutureless aortic bioprosthesis: fiveyear results of the $3 \mathrm{f}$ Enable long-term follow-up study. J Thorac Cardiovasc Surg 2014;148:1681-7.

7. Capelli C, Corsini C, Biscarini D, et al. PledgetArmed Sutures Affect the Haemodynamic Performance of Biologic Aortic Valve Substitutes: A Preliminary 
Experimental and Computational Study. Cardiovasc Eng Technol 2017;8:17-29.

8. Ensminger S, Fujita B, Bauer T, et al. Rapid Deployment Versus Conventional Bioprosthetic Valve Replacement for Aortic Stenosis. J Am Coll Cardiol 2018;71:1417-28.

9. The IVSSG Sutureless Projects. Available online: http:// suturelessprojects.com/

10. Chakravarty T, Søndergaard L, Friedman J, et al.

Cite this article as: Shuhaiber J. Technology for new surgical aortic valve replacement: current evidence and future directions. J Thorac Dis 2018;10(12):6392-6395. doi: 10.21037/ jtd.2018.11.25
Subclinical leaflet thrombosis in surgical and transcatheter bioprosthetic aortic valves: an observational study. Lancet 2017;389:2383-92.

11. Herrmann HC, Daneshvar SA, Fonarow GC, et al. Prosthesis-Patient Mismatch in 62,125 Patients Following Transcatheter Aortic Valve Replacement: From the STS/ ACC TVT Registry. J Am Coll Cardiol 2018;72:2701-11. 